\title{
ON THE RELATIONSHIP BETWEEN CONVERGENCE IN DISTRIBUTION AND CONVERGENCE OF EXPECTED EXTREMES
}

\author{
THEODORE P. HILL AND M. C. SPRUILL
}

(Communicated by Lawrence Gray)

\begin{abstract}
It is well known that the expected values $\left\{M_{k}(X)\right\}, k \geq 1$, of the $k$-maximal order statistics of an integrable random variable $X$ uniquely determine the distribution of $X$. The main result in this paper is that if $\left\{X_{n}\right\}$, $n \geq 1$, is a sequence of integrable random variables with $\lim _{n \rightarrow \infty} M_{k}\left(X_{n}\right)=\alpha_{k}$ for all $k \geq 1$, then there exists a random variable $X$ with $M_{k}(X)=\alpha_{k}$ for all $k \geq 1$ and $X_{n} \stackrel{\mathscr{L}}{\longrightarrow} X$ if and only if $\alpha_{k}=o(k)$, in which case the collection $\left\{X_{n}\right\}$ is also uniformly integrable. In addition, it is shown using a theorem of Müntz that any subsequence $\left\{M_{k_{j}}(X)\right\}, j \geq 1$, satisfying $\sum 1 / k_{j}=\infty$ uniquely determines the law of $X$.
\end{abstract}

\section{INTRODUCTION}

The distribution of a random variable $X$ is uniquely determined by its characteristic function $\phi_{X}$ and, if $X$ has finite mean, is also uniquely determined by the expected values of the $k$-maximal order statistics of $X$

$$
M_{k}(X):=E\left(\max _{1 \leq j \leq k} X_{j}\right), \quad X_{1}, X_{2}, \ldots, \text { i.i.d. } X_{j} \stackrel{\mathscr{L}}{=} X,
$$

a result due to Hoeffding (see [4]). For a sequence of random variables $\left\{X_{n}\right\}$ the classical continuity theorem says that if $\lim _{n \rightarrow \infty} \phi_{X_{n}}(t)=\phi(t)$ for all $t$, then a necessary and sufficient condition that $\phi$ be a characteristic function for some random variable $X$ (and that $X_{n} \stackrel{\mathscr{L}}{\longrightarrow} X$ ) is that $\phi$ be continuous at zero. The main result of this article is the following analog of the continuity theorem for the expected extremes $\left\{M_{k}\right\}$.

Theorem 1.1. Suppose $\left\{X_{n}\right\}, n \geq 1$, are integrable random variables such that $\lim _{n \rightarrow \infty} M_{k}\left(X_{n}\right)=\alpha_{k}$ exists and is finite for all $k \geq 1$. Then there exits $a$ random variable $X$ with $M_{k}(X)=\alpha_{k}$ for all $k \geq 1$ and $X_{n} \stackrel{\mathscr{L}}{\longrightarrow} X$ if and only if $\alpha_{k}=o(k)$, in which case $\left\{X_{n}\right\}$ is also uniformly integrable.

This note is organized as follows. Section 2 contains the proof of the main theorem. Section 3 contains an improvement of the Hoeffding result showing

Received by the editors March 17, 1992 and, in revised form, November 15, 1992.

1991 Mathematics Subject Classification. Primary 60F99; Secondary 60G70.

Key words and phrases. Convergence in distribution, convergence of expected extremes, maximal order statistics, Müntz's theorem, extreme value-theory. 
that the distribution of $X$ is determined by any subsequence $\left\{M_{k_{j}}(X)\right\}, j \geq 1$, satisfying $\sum 1 / k_{j}=\infty$. Section 4 contains applications to the asymptotic theory of extreme order statistics.

\section{Proof of MAIN THEOREM}

Throughout this note all random variables will be assumed to be integrable and defined on the same probability space $(\Omega, \mathscr{F}, P)$. Also $\mathbb{N}$ denotes the natural numbers; $E X$ the expectation of the random variable $X ; x \vee y$ and $x \wedge y$ the maximum and minimum of $x$ and $y$, respectively; $|x|$ the absolute value of $x ; X_{n} \stackrel{\mathscr{L}}{\longrightarrow} X$ convergence in distribution in the sense of [1]; $I(A)$ the indicator function of the set $A ; \delta(x)$ the Dirac (probability) measure with unit mass on $x$; and $F^{-1}$ the generalized (upper) inverse of the cumulative distribution function $F$, i.e.,

$$
F^{-1}(\beta)=\sup \{x: F(x) \leq \beta\} .
$$

For an integrable random variable $X$ and positive integer $k, \mathrm{~V}^{k} X$ and $\bigwedge_{k} X$ denote random variables with distributions of $\left(X_{1} \vee \cdots \vee X_{k}\right)$ and $\left(X_{1} \wedge \cdots \wedge X_{k}\right)$, respectively, where $X_{1}, X_{2}, \ldots$ are independent with the same distribution as $X$ (that is, $\mathrm{V}^{k} X$ is distributed as the $k$-maximal order statistic of $X$ and has distribution function $F^{k}$, where $F$ is the d.f. for $\left.X\right)$, and $M_{k}(X):=E\left(\bigvee^{k} X\right)$ and $m_{k}(X):=E\left(\bigwedge_{k} X\right)$. Observe that, since $E X$ is finite, so are $M_{k}(X)$ and $m_{k}(X)$ for all $k \in \mathbb{N}$, in contrast to the situation in classical moment theory where finiteness of the first moment does not imply finiteness of higher moments.

The proof of the next lemma is routine and left to the reader.

Lemma 2.1. (a) The following are equivalent:

(i) $\left\{X_{n}\right\}_{n \in \mathbb{N}}$ is uniformly integrable.

(ii) $\left\{\mathrm{V}^{k} X_{n}\right\}_{n \in \mathbb{N}}$ is uniformly integrable for all $k \in \mathbb{N}$.

(iii) $\left\{\bigwedge_{k} X_{n}\right\}_{n \in \mathbb{N}}$ is uniformly integrable for all $k \in \mathbb{N}$.

(b) The following are equivalent:

(i) $X_{n} \stackrel{\mathscr{L}}{\longrightarrow} X$.

(ii) $\bigvee^{k} X_{n} \stackrel{\mathscr{L}}{\longrightarrow} \bigvee^{k} X$ for some $k \in \mathbb{N}$.

(iii) $\left\{\bigvee^{k} X_{n} \stackrel{\mathscr{L}}{\longrightarrow} \bigvee^{k} X\right.$ for all $k \in \mathbb{N}$.

(iv) $\bigwedge_{k} X_{n} \stackrel{\mathscr{L}}{\longrightarrow} \bigwedge_{k} X$ for some $k \in \mathbb{N}$.

(v) $\bigwedge_{k} X_{n} \stackrel{\mathscr{L}}{\longrightarrow} \bigwedge_{k} X$ for all $k \in \mathbb{N}$.

Lemma 2.2. Suppose $X$ is an integrable random variable with distribution $F$. Then

(i) $M_{k}(X)=-\int_{-\infty}^{0} F^{k}(x) d x+\int_{0}^{\infty}\left(1-F^{k}(x)\right) d x=k \int_{0}^{1} F^{-1}(t) t^{k-1} d t$ for all $k \in \mathbb{N}$;

(ii) $\boldsymbol{M}_{k}(X)=\sum_{i=1}^{k}(-1)^{i+1}\left(\begin{array}{l}k \\ i\end{array}\right) m_{i}(X)$ and $m_{k}(X)=\sum_{i=1}^{k}(-1)^{i+1}\left(\begin{array}{l}k \\ i\end{array}\right) M_{i}(X)$ for all $k \in \mathbb{N}$

(iii) $M_{k}(X)=o(k)$ and $m_{k}=o(k)$; 
(iv) $M_{k+1}(X)-M_{k}(X) \leq M_{k}(X)-M_{k-1}(X)$ and $m_{k}(X)-m_{k+1}(X) \leq$ $m_{k-1}(X)-m_{k}(X)$ for all $k>1$, with equality throughout if and only if $X$ is degenerate;

(v) $Y \stackrel{\mathscr{L}}{=} X$ if and only if $M_{k}(Y)=M_{k}(X)$ for all $k \in \mathbb{N}$.

Proof. Conclusion (i) is a well-known consequence of integration by parts and Fubini's theorem. (ii) follows easily by the "inclusion-exclusion principle"

$$
\begin{gathered}
x_{1} \vee x_{2} \vee \cdots \vee x_{k}=x_{1}+\cdots+x_{k}-\sum_{i_{1} \neq i_{2}} x_{i_{1}} \wedge x_{i_{2}}+\sum_{i_{1}, i_{2}, i_{3} \text { distinct }} x_{i_{1}} \wedge x_{i_{2}} \wedge x_{i_{3}} \\
-\cdots \pm x_{1} \wedge x_{2} \wedge \cdots \wedge x_{k}
\end{gathered}
$$

and the fact that $X_{1}, X_{2}, \ldots$ (in the definitions of $M_{k}$ and $m_{k}$ ) are i.i.d. (iii) follows easily from (i) (in fact if $E|X|^{p}<\infty$, then $M_{k}(X)$ and $m_{k}(X)$ are both $o\left(k^{1 / p}\right)$; cf. $\left.[3,4]\right)$. (iv) is an easy exercise. (v) is Hoeffding's result.

The next lemma is the key tightness tool in the proof of the main theorem; its conclusion for arbitrary subsets $J \subseteq \mathbb{N}$ is used in $\S 3$.

Lemma 2.3. Suppose $E\left(X_{n}\right) \rightarrow \alpha$, where $-\infty<\alpha<\infty$. Then for all $J \subseteq \mathbb{N}$, if $\sup _{n \in J} M_{k}\left(X_{n}\right)<\infty$ (or $\left.\inf _{n \in J} m_{k}\left(X_{n}\right)>-\infty\right)$ for some $k \geq 2$, then all of the quantities $\sup _{n \in J} E\left|X_{n}\right|, \sup _{n \in J}\left|M_{2}\left(X_{n}\right)\right|$, and $\sup _{n \in J}\left|m_{2}\left(X_{n}\right)\right|$ are finite and the sequence $\left\{X_{n}\right\}_{n \in J}$ is tight.

Proof. If $\sup _{n \in J} M_{k}\left(X_{n}\right)<\infty$ for some $k \geq 2$, then also $\sup _{n \in J} M_{2}\left(X_{n}\right)<\infty$, since, for all $n, M_{2}\left(X_{n}\right) \leq M_{k}\left(X_{n}\right)$. Since $M_{1}\left(X_{n}\right) \rightarrow \alpha$ and since for each $n \in J$

$$
\int_{X_{n}>0} X_{n} d P+P\left(X_{n} \leq 0\right) E\left(X_{n}\right) \leq M_{2}\left(X_{n}\right),
$$

there exists a finite constant $K$ such that $E\left(X_{n}^{+}\right) \leq K+\sup _{n \in J} M_{2}\left(X_{n}\right)$. Therefore, since

$$
E\left(X_{n}\right)=E\left(X_{n}^{+}\right)-E\left(X_{n}^{-}\right) \rightarrow \alpha,
$$

it also follows that $\sup _{n \in J} E\left(X_{n}^{-}\right)<\infty$, so $\sup _{n \in J} E\left|X_{n}\right|<\infty$, which implies (by Markov's inequality) that $\left\{X_{n}\right\}_{n \in J}$ is tight. then

The proof will be completed once it is shown that $\inf _{n \in J} m_{2}\left(X_{n}\right)>-\infty$, for

$$
-\infty<\inf _{n \in J} m_{2}\left(X_{n}\right) \leq \sup _{n \in J} m_{2}\left(X_{n}\right) \leq \sup _{n \in J} M_{2}\left(X_{n}\right)<\infty .
$$

But, by Lemma 2.2(ii), $m_{2}\left(X_{n}\right)=2 E\left(X_{n}\right)-M_{2}\left(X_{n}\right)$, so

$$
\inf _{n \in J} m_{2}\left(X_{n}\right) \geq-2 \sup _{n \in J} E\left|X_{n}\right|-\sup _{n \in J} M_{2}\left(X_{n}\right)>-\infty .
$$

The hypothesis $k>1$ in Lemma 2.3 may not be weakened to $k \geq 1$, as can be seen by letting $k=1$ and considering $\left\{X_{n}\right\}$ satisfying $P\left(X_{n}=n\right)=\frac{1}{2}=$ $P\left(X_{n}=-n\right)$.

If $\sup _{k \in \mathbb{N}} \sup _{n \in J} M_{k}\left(X_{n}\right)<\infty$, then $\left\{X_{n}\right\}_{n \in J}$ is even uniformly bounded from above a.s. On the other hand, the hypothesis of Lemma 2.3 is not enough to imply uniform integrability, even for nonnegative random variables. 
Example 2.4. Let $\left\{X_{n}\right\}$ satisfy $P\left(X_{n}=n\right)=1 / n=1-P\left(X_{n}=0\right)$. Then $M_{k}\left(X_{n}\right)=n\left(1-(1-1 / n)^{k}\right) \rightarrow k$ as $n \rightarrow \infty$, so $\sup _{n} M_{k}\left(X_{n}\right)<\infty$ for each $k$, but $\left\{X_{n}\right\}$ is not u.i.

(This example also shows how in Theorem 1.1, if $\alpha_{k} \neq o(k)$, it can happen that $X_{n} \stackrel{\mathscr{L}}{\longrightarrow} X \equiv 0$, but not with $\alpha_{k}=M_{k}(X)$.)

Proof of Theorem 1.1. First suppose $\alpha_{k}=o(k)$. Lemma 2.3 (with $J=\mathbb{N}$ ) implies that $\sup _{n} E\left|X_{n}\right|<\infty$ and that $\left\{X_{n}\right\}$ is tight. It will now be shown that $\left\{X_{n}\right\}$ is uniformly integrable. Suppose, by way of contradiction, that

$$
\limsup _{\lambda \rightarrow \infty} \sup _{n} \int_{X_{n}>\lambda} X_{n} d P>0 .
$$

Then there exist sequences $\lambda_{m} \rightarrow \infty$ and $n_{m} \rightarrow \infty$ and $b>0$ satisfying

$$
\int_{X_{n_{m}}>\lambda_{m}} X_{n_{m}} d P=: b_{m}>b \text { for all } m \in \mathbb{N} \text {. }
$$

Let $p_{m}=P\left(X_{n_{m}}>\lambda_{m}\right)$; then $p_{m}>0$ by (2), since $b>0$. Since $\left\{X_{n}\right\}$ is tight and $\lambda_{m} \rightarrow \infty$,

$$
\lim _{m \rightarrow \infty} p_{m}=0 .
$$

For each $m \in \mathbb{N}$ let $c_{m}=b_{m} / p_{m}$ and $d_{m}=E\left[\left(X_{n_{m}}-b_{m}\right) /\left(1-p_{m}\right)\right]$ and define $Y_{m}$ by $P\left(Y_{m}=c_{m}\right)=p_{m}=1-P\left(Y_{m}=d_{m}\right)$. Observe that, for all $m$, $c_{m}>\lambda_{m} \geq d_{m}, E Y_{m}=E X_{n_{m}}$, and

$$
\left|d_{m}\right|<3 \sup _{n} E\left|X_{n}\right|=: L<\infty \text { for sufficiently large } m \text {. }
$$

By the conditional version of Jensen's Inequality,

$$
M_{k}\left(Y_{m}\right) \leq \dot{M}_{k}\left(X_{n_{m}}\right) \text { for all } k, m \in \mathbb{N} \text {. }
$$

Next calculate $M_{k}\left(Y_{m}\right)=c_{m}\left(1-\left(1-p_{m}\right)^{k}\right)+d_{m}\left(1-p_{m}\right)^{k}$, so by (2)-(4)

$$
\liminf _{m \rightarrow \infty} M_{k}\left(Y_{m}\right)>k b-L,
$$

which by (5) and the hypothesis $M_{k}\left(X_{n}\right) \rightarrow \alpha_{k}$ implies that

$$
\alpha_{k} \geq k b-L \text { for all } k \in \mathbb{N} \text {. }
$$

But since $b>0$, this contradicts the assumption that $\alpha_{k}$ is $o(k)$. Hence (1) does not hold, so $\lim \sup _{\lambda \rightarrow \infty} \sup _{n} \int_{X_{n}>\lambda} X_{n} d P \leq 0$. The argument to show $\geq 0$ is analogous, and together these imply that $\left\{X_{n}\right\}$ is uniformly integrable.

To show that $X_{n}$ converges in distribution, since $\left\{X_{n}\right\}$ is tight, it is enough to show (cf. [2, p. 346]) that if $\left\{X_{n_{m}}\right\}$ and $\left\{X_{n_{m^{\prime}}}\right\}$ are any subsequences with $X_{n_{m}} \stackrel{\mathscr{L}}{\longrightarrow} X$ and $X_{n_{m^{\prime}}} \stackrel{\mathscr{L}}{\longrightarrow} Y$, then $X \stackrel{\mathscr{L}}{=} Y$. By Lemma $2.2(\mathrm{v})$ it suffices to show that

$$
M_{k}(X)=M_{k}(Y) \text { for all } k \in \mathbb{N} \text {. }
$$

For fixed $k$ Lemma 2.1(a) implies that $\left\{\bigvee^{k} X_{n}\right\}_{n \in \mathbb{N}}$ is uniformly integrable (and hence that $\left\{\mathrm{V}^{k} X_{n_{m}}\right\}$ and $\left\{\mathrm{V}^{k} X_{n_{m^{\prime}}}\right\}$ are uniformly integrable) and Lemma 2.1(b) implies that $\mathrm{V}^{k} X_{n_{m}} \stackrel{\mathscr{L}}{\longrightarrow} \mathrm{V}^{k} X$ and $\mathrm{V}^{k} X_{n_{m^{\prime}}} \stackrel{\mathscr{L}}{\longrightarrow} \mathrm{V}^{k} Y$, so (by [1, Theorem 5.4, p. 32]) $M_{k}(X)$ and $M_{k}(Y)$ are both finite and $\lim _{m \rightarrow \infty} M_{k}\left(X_{n_{m}}\right)=$ 
$M_{k}(X)$ and $\lim _{m^{\prime} \rightarrow \infty} M_{k}\left(X_{n_{m^{\prime}}}\right)=M_{k}(Y)$. Since $\lim _{n \rightarrow \infty} M_{k}\left(X_{n}\right)=\alpha_{k}$, this establishes (6) and shows that $M_{k}(X)=\alpha_{k}$ for all $k$.

Conversely, suppose there exists a random variable $X$ with $M_{k}(X)=\alpha_{k}$ for all $k \geq 1$ and $X_{n} \stackrel{\mathscr{L}}{\longrightarrow} X$. Since $E X=M_{1}(X)=\alpha_{1}$ is finite, $\alpha_{k}$ is $o(k)$ by Lemma 2.2(iii), and uniform integrability of the $\left\{X_{n}\right\}$ follows as before.

\section{Determinacy of MÜNTZ SUbSequenCes OF EXPECTED EXTREMES}

The main purpose of this section is to use a theorem of Müntz to prove the following improvement of Hoeffding's result Lemma 2.2(v): for a different strengthening, showing that sequences of expectations of nonextreme order statistics also suffices to determine the distribution, see [9]. In [4] this notion of determination of a distribution by its expected extremes is generalized in defining a partial order $\leq_{e}$ on the class of finite-mean distributions by $F_{X} \leq_{e} F_{Y}$ iff $M_{k}(X) \leq M_{k}(Y)$ for all $k$.

Theorem 3.1. For integrable random variables $X$ and $Y$ the following are equivalent:

(i) $X \stackrel{\mathscr{L}}{=} Y$.

(ii) $M_{k_{j}}(X)=M_{k_{j}}(Y)$ for all $j$, for some distinct $\left\{k_{j}\right\} \subseteq \mathbb{N}$ with $\sum 1 / k_{j}=$ $\infty$.

(iii) $M_{k}(X)=M_{k}(Y)$ for all $k \in \mathbb{N}$.

(iv) $m_{k_{j}}(X)=m_{k_{j}}(Y)$ for all $j$, for some distinct $\left\{k_{j}\right\} \subseteq \mathbb{N}$ with $\sum 1 / k_{j}=$ $\infty$

(v) $m_{k}(X)=m_{k}(Y)$ for all $k \in \mathbb{N}$.

Proof. To see (ii) $\Rightarrow$ (i), fix positive integers $k_{1}<k_{2}<\cdots$ satisfying $\sum 1 / k_{j}=$ $\infty$ and suppose that $M_{k_{j}}(X)=M_{k_{j}}(Y)$ for all $j \geq 1$, where $X$ and $Y$ are integrable random variables with distributions $F$ and $G$, respectively. By Lemma 2.2(i), $\int_{0}^{1}\left|F^{-1}(t)\right| d t$ and $\int_{0}^{1}\left|G^{-1}(t)\right| d t$ are both finite and

$$
\int_{0}^{1} t^{k_{j}-1} F^{-1}(t) d t=\int_{0}^{1} t^{k_{j}-1} G^{-1}(t) d t \quad \text { for all } j \geq 1 .
$$

Let $f$ and $g$ be the $L_{1}[0,1]$ functions defined by $f(t)=t^{k_{1}-1} F^{-1}(t)$ and $g(t)=t^{k_{1}-1} G^{-1}(t)$, and define the signed measures $\mu$ and $\nu$ by $\mu([0, t])=$ $\int_{0}^{t} f(x) d x$ and $\nu([0, t])=\int_{0}^{t} g(x) d x$. Then

$$
\int_{0}^{1} t^{k_{j}-k_{1}} d(\mu(t)-\nu(t))=0 \text { for all } j \geq 1 .
$$

Thus the bounded linear functional $\Delta$ on $C[0,1]$ defined by $\Delta(\phi)=$ $\int_{0}^{1} \phi(t) d(\mu(t)-\nu(t))$ vanishes on the set of functions $t^{k_{j}-k_{1}}$ for all $j \geq 1$. By Müntz's theorem (cf. [7]), since $\sum_{j>1} 1 /\left(k_{j}-k_{1}\right)=\infty$, the collection $\left\{t^{k_{j}-k_{1}}\right\}_{j \geq 1}$ has dense linear span in $C[0,1]$, so $\Delta$ is the zero functional and $\mu=\nu$. Taking Radon-Nikodym derivatives, this implies $F^{-1}=G^{-1}$ for almost all $t \in[0,1]$, and this implies that $F=G$.

Clearly (i) $\Rightarrow$ (iii) $\Rightarrow$ (ii), and conclusions (iv) and (v) follow similarly. 
Remark. Müntz's theorem states that the divergence of $\sum 1 / k_{j}$ is also a necessary condition for the functions $\left\{t^{k_{j}}\right\}$ to be dense in $C[0,1]$. An analogous statement is also true here, but this is not needed for the results in this paper. If $\sum 1 / k_{j}<\infty$, then there are distinct random variables $X$ and $Y$ such that $M_{k_{j}}(X)=M_{k_{j}}(Y)$ for all $j$.

The combined results of Theorems 1.1 and 3.1 may be summarized as follows.

Theorem 3.2. For all sequences of integrable random variables $\left\{X_{n}\right\}_{n \in \mathbb{N}}$ the following are equivalent :

(i) $\lim _{n \rightarrow \infty} M_{k_{j}}\left(X_{n}\right)=\alpha_{k_{j}}$ exists and is finite for all $j$, for some distinct $\left\{k_{j}\right\} \subseteq \mathbb{N}$ with $k_{1}=1$, and $\sum 1 / k_{j}=\infty$ and $\alpha_{k_{j}}=o\left(k_{j}\right)$.

(ii) $\lim _{n \rightarrow \infty} M_{k}\left(X_{n}\right)=\alpha_{k}$ exists and is finite for all $k \in \mathbb{N}$ and $\alpha_{k}=o(k)$.

(iii) $\lim _{n \rightarrow \infty} m_{k_{j}}\left(X_{n}\right)=\beta_{k_{j}}$ exists and is finite for all $j$, for some distinct $\left\{k_{j}\right\} \subseteq \mathbb{N}$ with $k_{1}=1$, and $\sum 1 / k_{j}=\infty$ and $\beta_{k_{j}}=o\left(k_{j}\right)$.

(iv) $\lim _{n \rightarrow \infty} m_{k}\left(X_{n}\right)=\beta_{k}$ exists and is finite for all $k \in \mathbb{N}$ and $\beta_{k}=o(k)$.

(v) There exists an integrable random variable $X$ such that $X_{n} \stackrel{\mathscr{L}}{\longrightarrow} X$ and $\lim _{n \rightarrow \infty} E\left|X_{n}\right|=E|X|$.

(vi) There exists an integrable random variable $X$ such that $X_{n} \stackrel{\mathscr{L}}{\longrightarrow} X$ and $\left\{X_{n}\right\}_{n \in \mathbb{N}}$ is uniformly integrable.

The determinancy results Theorem 3.1(ii) and (iv) allow $k_{1}$ to be arbitrary, whereas in the convergence results Theorem 3.2(i) and (iii) the requirement that $k_{1}=1$ cannot be dropped, essentially since the conclusion of Lemma 2.3 may then fail.

Example 3.3. Let $\left\{X_{n}\right\}$ satisfy $P\left(X_{n}=-n\right)=n^{-2 / 3}=1-P\left(X_{n}=0\right)$. Then $M_{1}\left(X_{n}\right)=E X_{n} \rightarrow-\infty, M_{k}\left(X_{n}\right) \rightarrow 0$ for $k>1$ and $X_{n} \stackrel{\mathscr{L}}{\longrightarrow} 0$ (so $\sup _{n} E\left|X_{n}\right|=\infty$ and $\left.M_{1}\left(X_{n}\right) \nrightarrow M_{1}(X)\right)$.

In the other direction, if $X_{n} \stackrel{\mathscr{L}}{\longrightarrow} X$, the hypotheses $E\left|X_{n}\right| \rightarrow E|X|$ or $\left\{X_{n}\right\}$ u.i. may not be weakened to $E X_{n} \rightarrow E X$ and $\left\{X_{n}\right\}$ tight, as the following example shows.

Example 3.4. Let $\left\{X_{n}\right\}$ satisfy $P\left(X_{n}=0\right)=1-1 / n$ and $P\left(X_{n}=n^{3 / 2}\right)=$ $P\left(X_{n}=-n^{3 / 2}\right)=\frac{1}{2} n$, and let $P(X=0)=1$. Then $X_{n} \stackrel{\mathscr{L}}{\longrightarrow} X$ and $E X_{n}=0=$ $E X$ for all $n$, and $\left\{X_{n}\right\}$ is tight, but $M_{2}\left(X_{n}\right)=\sqrt{n}-(2 \sqrt{n})^{-1} \rightarrow \infty$, while $M_{2}(X)=0$.

\section{Applications}

Theorem 1.1 allows convergence in distribution to be demonstrated purely by analyzing the "maximal moments" $\left\{M_{k}\right\}$. For example, to show $X_{n} \stackrel{\mathscr{L}}{\longrightarrow}$ $U[0,1]$, it is sufficient to show that $M_{k}\left(X_{n}\right) \rightarrow k /(k+1)$ for all $k$ (or in fact, just for a "Müntz subsequence" $\left.\left\{k_{j}\right\}\right)$. Similarly, to show central limit theorem results, the problem is to show convergence of the expected extremes to those for, say, a $N(0,1)$ random variable. In the case of convergence to a constant 
$X \equiv \mu$, the criteria $M_{k}\left(X_{n}\right) \rightarrow M_{k}(X)$ simply reduces to showing $M_{k}\left(X_{n}\right) \rightarrow \mu$ and Theorem 1.1 can be used to give an alternative combinatorial-based proof of the weak and $L_{1}$ law of large numbers.

The existential conclusion of Theorem 1.1 can be applied to the asymptotic theory of extreme order statistics as in [5]. Let $X_{1}, X_{2}, \ldots$ be i.i.d. with c.d.f. $F$, let $Z_{n}=\max \left\{X_{1}, \ldots, X_{n}\right\}$, and let $Y_{n}=g_{n}\left(Z_{n}\right)$, where $g_{n}: \mathbb{R} \rightarrow \mathbb{R}$ is monotone increasing. A basic question in this theory is: Under what conditions on $F$ and $\left\{g_{n}\right\}$ does $Y_{n} \stackrel{\mathscr{L}}{\longrightarrow} Y$ for some random variable $Y$ ? The standard classical setting (e.g., [5]) is the linear case $g_{n}(x)=\left(x-a_{n}\right) / b_{n}$, but the maximal moment method applies equally well to general $g_{n}$. By Theorem 1.1 a sufficient condition for the existence of such a $Y$ is that $M_{k}\left(Y_{n}\right) \rightarrow \alpha_{k}$ for some finite $\left\{\alpha_{k}\right\}$ satisfying $\alpha_{k}=o(k)$. By the monotonicity of $g_{n}$ and the i.i.d.-ness of the $\left\{X_{j}\right\}$,

$$
M_{k}\left(Y_{n}\right)=E\left[g_{n}\left(Z_{n k}\right)\right] .
$$

In the classical setting, if $F$ is the uniform distribution on $(0,1)$ and $g_{n}(x)=n(x-1)$, then $M_{k}\left(Y_{n}\right)=n E\left[Z_{n k}-1\right]=-n /(n k+1) \rightarrow-1 / k=M_{k}(Y)$, where $Y$ has c.d.f. $e^{x}$ for $x \leq 0$. Thus Theorem 1.1 implies that

$$
n\left(Z_{n}-1\right) \stackrel{\mathscr{L}}{\longrightarrow} Y
$$

(cf. [5, pp. 64-65]).

For the uniform distribution again, taking nonlinear $g_{n}=n\left(x^{2}-1\right)$, the analogous implication of Theorem 1.1 is

$$
n\left(Z_{n}^{2}-1\right) \stackrel{\mathscr{L}}{\longrightarrow} 2 Y \text {. }
$$

Since $Z_{n k} \stackrel{\mathscr{L}}{=} F^{-1}\left(U_{n k}\right)$, where $U_{n k}$ has c.d.f. $H(t)=t^{n k}, 0<t<1$, a change of variables implies that

$$
M_{k}\left(Y_{n}\right)=\int_{0}^{1} g_{n} \circ F^{-1}(u) n k u^{n k-1} d u=\int_{0}^{1} g_{n} \circ F^{-1}\left(t^{1 / n}\right) k t^{k-1} d t
$$

(from which it can readily be seen that the $o(k)$ property of the $\left\{\alpha_{k}\right\}$ depends heavily on the values of $F^{-1}$ near 1 ; that is, it is essentially a tail property). Thus if $g_{n} \circ F^{-1}\left(t^{1 / n}\right)$ converges nicely to an increasing $h(t)$, then

$$
M_{k}\left(Y_{n}\right) \rightarrow \int_{0}^{1} h(t) k t^{k-1} d t=E\left[h\left(U_{k}\right)\right]
$$

Using this observation, Theorem 1.1 can be applied both to general nonlinear $g_{n}$ 's (e.g., $g_{n}=F^{n}$ shows the well-known fact that $F^{n}\left(Z_{n}\right)$ is uniform on $(0,1))$ and to the classical linear case to also yield facts about convergence of expected values as in the following proposition (cf. [8]). In this application $M_{k}\left(Y_{n}\right)$ cannot be calculated explicitly, since $F$ is not specified exactly.

Proposition 4.1. Let $F$ be the c.d.f. of a random variable $X$ such that $E\left(X^{-}\right)<$ $\infty$, ess $\sup X=\infty$, and for some $\gamma>0$

$$
\lim _{t \rightarrow \infty} \frac{1-F(x t)}{1-F(t)}=x^{-\gamma} \text { for all } x>0 .
$$


Then $Z_{n} / F^{-1}(1-1 / n)$ converges in distribution and is uniformly integrable if and only if $\gamma>1$. If $\gamma>1$, the limiting distribution is $H_{\gamma}(x)=e^{-x^{-\gamma}}$ for $x>0$.

Proof. Let $Y_{n}=Z_{n} / F^{-1}(1-1 / n)$, and set $h_{n}(t)=F^{-1}\left(t^{1 / n}\right) / F^{-1}(1-1 / n)$.

By (8) and ess sup $X=+\infty$,

$$
\lim _{n \rightarrow \infty} h_{n}(t)=(-\ln t)^{-1 / \gamma}
$$

By (7) (with $g_{n}(x)=x / F^{-1}(1-1 / n)$ and $\left.k=1\right)$, (9), and Fatou's lemma,

$$
\lim \inf E\left|Y_{n}\right| \geq \liminf E Y_{n} \geq \int_{0}^{1}(-\ln t)^{-1 / \gamma} d t .
$$

This last integral is infinite if and only if $\gamma \leq 1$, so for $\gamma \leq 1,\left\{Y_{n}\right\}$ is not uniformly integrable.

Observe by Lemma 2.2 (i) that $M_{k}\left(H_{\gamma}\right)=\int_{0}^{1}(-\ln t)^{-1 / \gamma} k t^{k-1} d t<\infty$ for $\gamma>1$. By Theorem 1.1, to complete the proof of the proposition it suffices to show that for $\gamma>1$

$$
\lim _{n \rightarrow \infty} \int_{0}^{1} h_{n}(t) k t^{k-1} d t=\int_{0}^{1}(-\ln t)^{-1 / \gamma} k t^{k-1} d t .
$$

Set $p=F(0)<1$, and observe first that since $E\left[X^{-}\right]=\int_{0}^{p}\left|F^{-1}(u)\right| d u<\infty$,

$$
\left|\int_{0}^{p^{n}} h_{n}(t) k t^{k-1} d t\right| \leq \frac{n k p^{n k-1}}{F^{-1}(1-1 / n)} \int_{0}^{p}\left|F^{-1}(u)\right| d u \rightarrow 0 \quad \text { as } n \rightarrow \infty .
$$

Since the functions $h_{n}(t) k t^{k-1} I_{\left(p^{n}, \delta\right)}(t)$ are uniformly bounded and converge to $(-\ln t)^{-1 / \gamma} k t^{k-1} I_{(0, \delta)}(t)$, bounded convergence implies

$$
\int_{p^{n}}^{\delta} h_{n}(t) k t^{k-1} d t \rightarrow \int_{0}^{\delta}(-\ln t)^{-1 / \gamma} k t^{k-1} d t \quad \text { for all } \delta<1 .
$$

Finally, to check that

$$
\lim _{\delta \rightarrow 1} \lim _{n \rightarrow \infty}\left|\int_{\delta}^{1} h_{n}(t) k t^{k-1} d t\right|=0
$$

use the representation (see [5, Appendix III])

$$
L(x)=u(x) \exp \left\{\int_{B}^{x} \frac{e(y)}{y} d y\right\}
$$

of the slowly varying function $L(x)=x^{\gamma}(1-F(x))$ to show that for $\delta$ sufficiently close to 1 there exist constants $K$ and $c, \gamma<c<1$, such that for sufficiently large $n$

$$
h_{n}(t) \leq K\left[n\left(1-t^{1 / n}\right)\right]^{-1 / c} \quad \text { for all } t \in(\delta, 1) .
$$

Equation (10) follows from (11)-(13).

\section{ACKNOWLEDGEMENT}

The authors are grateful to Professors Richard Duke, Jeffrey Geronimo, and David Gilat for several useful conversations, to Professor Olav Kallenberg for pointing out an error in an earlier draft, and especially to the referee for suggesting the final version of the main theorem. 


\section{REFERENCES}

1. P. Billingsley, Convergence of probability measures, Wiley, New York, 1968.

2. ___ Probability and measure, 2nd ed., Wiley, New York, 1986.

3. P. Downey, Distribution-free bounds on the expectation of the maximum, with scheduling applications, Oper. Res. Lett. 9 (1990), 189-201.

4. P. Downey and R. Maier, Orderings arising from expected extremes, with an application, IMS Lecture Notes-Monograph Ser., vol. 22, Inst. Math. Statist., Hayward, CA, 1992, pp. 66-75.

5. J. Galambos, The asymptotic theory of extreme order statistics, Wiley, New York, 1978.

6. C. Müntz, Über den approximationssatz von Weierstrass, Schwartz-Festschrift, 1914.

7. I. Natanson, Constructive function theory, Vol. II, Fredrick Ungar, New York, 1965.

8. J. Pickands, Moment convergence of sample extremes, Ann. Math. Statist. 39 (1968), 881889.

9. M. Pollack, On equal distributions, Ann. Statist. 1 (1973), 180-182.

School of Mathematics, Georgia Institute of Technology, Atlanta, Georgia 30332

E-mail address: spruill@math.gatech.edu 